sensitivity and $70 \%$ specificity. A subset of 8 of these was found to be at least as accurate as the 20-transcript set. A patent application has been lodged in relation to these two sets of transcripts.

Genetic profiling of peripheral blood mononuclear cells is a noninvasive procedure that has the potential to direct choice of RA therapy. It should be remembered, however, that the model devised by Lequerré and colleagues applies only to patients with long-standing and very active RA. Similar studies in patients with recent-onset or moderate disease are recommended.

Original article Lequerré T et al. (2006) Gene profiling in white blood cells predicts infliximab responsiveness in rheumatoid arthritis. Arthritis Res Ther 8: R105

\section{High initial levels of comorbidity in patients with RA selected to receive biologic agents}

Although the pivotal, randomized, controlled trials of biologic therapies for rheumatoid arthritis (RA) did not show an increase in serious adverse events in biologic-agent-treated patients compared with placebo-treated patients, there has been concern about the safety of biologic agents in routine practice, because those trials excluded patients with substantial comorbidity. Indeed, there have been reports of increases in serious adverse events in patients who receive biologic agents; however, before these events can be attributed to the new treatments, the baseline comorbidity of patients selected for biologic treatment should ascertained. British investigators, therefore, carried out a national, prospective, observational study to identify the prevalence and types of comorbidity in RA patients treated with biologic agents.

Data on patients with RA were obtained from the British Society for Rheumatology Biologics Register. Analysis of records on the 7,818 patients registered before 1 October 2004 revealed that 3,302 received etanercept, 3,332 received infliximab, 1,059 received adalimumab, and 132 received anakinra. At the time of registration, $58 \%$ of patients had at least one comorbid condition, and $25 \%$ had more than one comorbid condition. The most frequent comorbidities were cardiovascular disease, respiratory disease, and depression. Serious adverse events that occur after the initiation of biologic therapy should, therefore, be interpreted in light of the high baseline levels of comorbidity in this population.

Original article Hyrich K et al. (2006) Baseline comorbidity levels in biologic and standard DMARD treated patients with rheumatoid arthritis: results from a national patient register. Ann Rheum Dis 65: 895-898

\section{Alendronate limits the effectiveness of teriparatide in combination therapy}

Alendronate and teriparatide both improve BMD in osteoporotic patients, and it seems logical that combination therapy with both agents would have a higher efficacy than either of them alone. When this combination therapy was used in two randomized, controlled trials (one by Finkelstein et al.), researchers made the surprising discovery that combination therapy was, at best, no more effective than teriparatide alone at increasing BMD. Alendronate even seemed to impair teriparatide's efficacy. Finkelstein et al. further analyzed the data from their trial in order to determine whether alendronate inhibits teriparatide's stimulatory effect on bone turnover.

The analysis included data from 63 osteoporotic men, who had received either alendronate $10 \mathrm{mg}$ daily, teriparatide $37 \mu \mathrm{g}$ daily (from month 6), or both, for 30 months. Serum levels of the bone-turnover markers $\mathrm{N}$-telopeptide, osteocalcin, and amino-terminal propeptide of type 1 procollagen were regularly assessed. In the alendronate group, marker levels initially declined and then remained stable. In the teriparatide group, levels of all three markers increased during the first 6 months of treatment, then decreased towards baseline over the next 18 months. In the combination group, marker levels were higher than those seen with alendronate monotherapy, but much lower than those seen with teriparatide monotherapy.

The authors note that $\mathrm{N}$-telopeptide levels increased slightly in the combination group when teriparatide was added; this finding suggests that alendronate does not completely block teriparatide's stimulation of bone resorption. They conclude that alendronate does, however, markedly impair teriparatide's capacity to increase bone turnover.

Original article Finkelstein JS et al. (2006) Effects of teriparatide, alendronate, or both on bone turnover in osteoporotic men. J Clin Endocrinol Metab

[doi: 10.1210/jc.2006-0190] 